Volume 134, Number 1, Pages 259-263

S 0002-9939(05)07988-8

Article electronically published on June 14, 2005

\title{
ZERO SETS AND INTERPOLATING SETS IN FOCK SPACES
}

\author{
JAMES TUNG
}

(Communicated by Juha M. Heinonen)

\begin{abstract}
An example is constructed to show that interpolating sets for Fock spaces are not necessarily zero sets.
\end{abstract}

\section{INTRODUCTION}

For $1 \leq p<\infty$ and $\alpha>0$, the Fock space $F_{\alpha}^{p}$ consists of all entire functions $f$ for which

$$
\|f\|_{p, \alpha}^{p}=\int_{\mathbb{C}}\left|f(z) e^{-\frac{\alpha}{2}|z|^{2}}\right|^{p} d A(z)<\infty .
$$

The space $F_{\alpha}^{\infty}$ consists of all entire functions $f$ for which

$$
\|f\|_{\infty, \alpha}=\sup _{z \in \mathbb{C}}|f(z)| e^{-\frac{\alpha}{2}|z|^{2}}<\infty .
$$

A sequence of distinct complex numbers $\left\{z_{j}\right\}$ is called an interpolating set for $F_{\alpha}^{p}$ if for every sequence $\left\{a_{j}\right\}$ with the property

$$
\sum_{j=1}^{\infty}\left|a_{j} e^{-\frac{\alpha}{2}\left|z_{j}\right|^{2}}\right|^{p}<\infty
$$

there is a function $f \in F_{\alpha}^{p}$ solving the interpolation problem

$$
f\left(z_{j}\right)=a_{j}, \quad j=1,2, \ldots
$$

A sequence $\Gamma=\left\{z_{j}\right\}$ is said to be uniformly discrete if there is a constant $\delta>0$ such that

$$
\left|z_{j}-z_{k}\right|>\delta, \quad j \neq k
$$

The supremum of all such $\delta$ is called the separation constant of $\Gamma$. For $\zeta \in \mathbb{C}$ and $r>0$, let $B(\zeta, r)$ denote the disk $|z-\zeta|<r$. Let $n(\Gamma, \zeta, r)$ be the number of points of $\Gamma$ that lie in $B(\zeta, r)$. The upper uniform density of $\Gamma$ is

$$
D^{+}(\Gamma)=\limsup _{r \rightarrow \infty} \sup _{\zeta \in \mathbb{C}} \frac{n(\Gamma, \zeta, r)}{r^{2}} .
$$

Received by the editors August 2, 2004 and, in revised form, September 3, 2004.

2000 Mathematics Subject Classification. Primary 30E05; Secondary 46E15.

Key words and phrases. Entire functions, interpolating sequences, zero sets.

This paper is part of the author's dissertation at the University of Michigan under the direction of Professor Peter Duren. The author also thanks Joaquim Ortega-Cerdà for helpful discussions. 
Seip and Wallstén [4, 5] characterized interpolating sets in terms of their upper uniform densities, as follows.

Theorem (Seip-Wallstén). Let $1 \leq p<\infty$ and $\alpha>0$. A complex sequence $\Gamma$ is an interpolating set for $F_{\alpha}^{p}$ if and only if it is uniformly discrete and $D^{+}(\Gamma)<\alpha$.

The theorem shows that for a fixed $\alpha$, the interpolating sets for $F_{\alpha}^{p}$ are independent of $p$. Qualitatively, an interpolating set must be sparse in $\mathbb{C}$.

A set $\Gamma=\left\{z_{j}\right\}$ is called a zero set of a space $X$ of analytic functions if there is a function $f \in X$ whose zeros are precisely $\Gamma$, with the usual convention that repeated elements of $\Gamma$ are zeros of prescribed order. The Bergman space $A^{p}$ consists of those functions $f$ analytic in the unit disk $\mathbb{D}$ such that

$$
\int_{\mathbb{D}}|f(z)|^{p} d A(z)<\infty .
$$

Horowitz [2] showed that every subset of an $A^{p}$ zero set is also an $A^{p}$ zero set (see also Chapter 4 of [3]). However, Zhu [6] showed that a subset of an $F_{\alpha}^{p}$ zero set need not be an $F_{\alpha}^{p}$ zero set.

A sequence of distinct points $\left\{z_{j}\right\}$ in the disk $\mathbb{D}$ is called an interpolating set for $A^{p}$ if for every sequence $\left\{a_{j}\right\}$ with

$$
\sum_{j=1}^{\infty}\left|a_{j}\right|^{p}\left(1-\left|z_{j}\right|^{2}\right)^{2}<\infty,
$$

there is a function $f \in A^{p}$ solving the interpolation problem

$$
f\left(z_{j}\right)=a_{j}, \quad j=1,2, \ldots
$$

It is easy to show that interpolating sets for $A^{p}$ are also $A^{p}$ zero sets. Given an interpolating set $\left\{z_{j}\right\}$ for $A^{p}$, let $f \in A^{p}$ be a solution to

$$
f\left(z_{1}\right)=1, \quad f\left(z_{j}\right)=0 \quad \text { for } j \geq 2 .
$$

Then the non-zero function $\left(z-z_{1}\right) f(z)$ belongs to $A^{p}$ and vanishes on all $z_{j}$. Now Horowitz's result quoted above allows us to conclude that there is some function in $A^{p}$ vanishing precisely on the set $\left\{z_{j}\right\}$.

The corresponding argument fails in the Fock space on two accounts. The factor $\left(z-z_{1}\right)$ is unbounded in $\mathbb{C}$, so for $f \in F_{\alpha}^{p}$ the function $\left(z-z_{1}\right) f(z)$ need not belong to $F_{\alpha}^{p}$. Also, in view of Zhu's result, a non-zero function in $F_{\alpha}^{p}$ may vanish on a set $\Gamma$ without $\Gamma$ being an $F_{\alpha}^{p}$ zero set. In fact, the corresponding conclusion fails as well. In the next section of the paper we will show that, contrary to the situation in Bergman spaces, an interpolating set for $F_{\alpha}^{p}$ need not be an $F_{\alpha}^{p}$ zero set.

\section{An interpolating SET that IS NOt A ZERO SET}

Before giving the construction, we recall some background from the theory of entire functions. Proofs can be found, for instance, in Boas 1 .

Let $f$ be an entire function, and let $M(r, f)=\max _{|z|=r}|f(z)|$. The order of $f$ is

$$
\rho=\limsup _{r \rightarrow \infty} \frac{\log \log M(r, f)}{\log r} .
$$


If $0<\rho<\infty$, the type of $f$ is

$$
\tau=\limsup _{r \rightarrow \infty} \frac{\log M(r, f)}{r^{\rho}} .
$$

It is clear from the above definition that if a function $f$ has order $\rho$, then for every $\varepsilon>0$,

$$
M(r, f)=O\left(e^{r^{\rho+\varepsilon}}\right), \quad r \rightarrow \infty .
$$

Thus any function with order $\rho<2$ must be in $F_{\alpha}^{p}$. Conversely, we can use the fact that point-evaluation functionals are bounded to determine the order and type of a function in the Fock space fairly precisely; see Theorem 2 of Zhu 6 .

Lemma 1. Every function $f \in F_{\alpha}^{p}$ has order $\rho \leq 2$. If $\rho=2$, then $f$ has type $\tau \leq \frac{\alpha}{2}$.

For any entire function $f$, let its zeros $z_{j}$ be enumerated so that $\left|z_{1}\right| \leq\left|z_{2}\right| \leq \ldots$. The convergence exponent of $\left\{z_{j}\right\}$ is the infimum $\rho_{1}$ of the positive numbers $\beta$ for which

$$
\sum_{j} \frac{1}{\left|z_{j}\right|^{\beta}}<\infty
$$

Let $n(r)$ be the number of zeros of $f$ inside the disk $B(0, r)$. If $f$ has order $\rho$ with $0<\rho<\infty$, let

$$
S(r)=\sum_{0<\left|z_{j}\right|<r} \frac{1}{\left(z_{j}\right)^{\rho}} .
$$

The following lemma is a well-known consequence of Jensen's theorem.

Lemma 2. Let $f$ be an entire function of order $\rho$, and let $\rho_{1}$ be the convergence exponent of its zeros. Then $\rho_{1} \leq \rho$.

One can show, via the Hadamard factorization theorem, that an entire function with non-integer order $\rho$ has finite type if and only if $n(r)=O\left(r^{\rho}\right)$. If $\rho$ is an integer, there is an additional restriction. The following theorem is due to Lindelöf (see Boas [1], p. 27).

Theorem (Lindelöf). If the order of an entire function $f$ is a positive integer $\rho$, then $f$ is of finite type if and only if both $n(r)=O\left(r^{\rho}\right)$ and the sums $S(r)$ are bounded.

Lindelöf's theorem will be the key to our construction.

Theorem. An interpolating set in the Fock space $F_{\alpha}^{p}$ need not be an $F_{\alpha}^{p}$ zero set.

Proof. Let $\delta>0$. For each integer $k \geq 1$, let $\Gamma_{k}$ be the set of $(k+1)$ points evenly spaced in the first quadrant on the circle $|z|=k \delta$, including the points $k \delta$ and $i k \delta$. Let $\Gamma=\bigcup_{k=1}^{\infty} \Gamma_{k}$, and write $\Gamma=\left\{z_{j}\right\}$.

First we observe that the distance between two neighboring points in $\Gamma_{k}$ is $2 k \delta \sin \frac{\pi}{4 k}>\delta$, so we may choose $\delta>\frac{2}{\sqrt{\alpha}}$ to ensure that $\Gamma$ is uniformly discrete with a separation constant larger than $\frac{2}{\sqrt{\alpha}}$. We claim that the resulting set $\Gamma$ is an interpolating set for $F_{\alpha}^{p}$. Indeed, if the set $\left\{z_{j}\right\}$ is uniformly discrete with separation constant $\delta$, then the disks $B\left(z_{j}, \frac{\delta}{2}\right)$ are pairwise disjoint. Given any $\zeta \in \mathbb{C}$ and 
$r>0$, if $z_{j} \in B(\zeta, r)$, the triangle inequality implies that $B\left(z_{j}, \frac{\delta}{2}\right) \subset B\left(\zeta, r+\frac{\delta}{2}\right)$. Therefore,

$$
\bigcup_{z_{j} \in B(\zeta, r)} B\left(z_{j}, \frac{\delta}{2}\right) \subset B\left(\zeta, r+\frac{\delta}{2}\right) .
$$

A comparison of areas now shows that

$$
\pi\left(\frac{\delta}{2}\right)^{2} n(\Gamma, \zeta, r) \leq \pi\left(r+\frac{\delta}{2}\right)^{2}
$$

Thus $n(\Gamma, \zeta, r)$ has an upper bound independent of the center $\zeta$, so it follows that

$$
D^{+}(\Gamma)=\limsup _{r \rightarrow \infty} \sup _{\zeta \in \mathbb{C}} \frac{n(\Gamma, \zeta, r)}{r^{2}} \leq \limsup _{r \rightarrow \infty} \frac{4\left(r+\frac{\delta}{2}\right)^{2}}{\delta^{2} r^{2}}=\frac{4}{\delta^{2}} .
$$

Thus the choice $\delta>\frac{2}{\sqrt{\alpha}}$ implies $D^{+}(\Gamma)<\alpha$, so by the Seip-Wallstén theorem, $\Gamma$ is an interpolating set for $F_{\alpha}^{p}$.

Now suppose for purpose of contradiction that $\Gamma$ is the zero set of some function $f \in F_{\alpha}^{p}$. Observe that $\Gamma$ has the property

$$
\sum_{j=1}^{\infty} \frac{1}{\left|z_{j}\right|^{\beta}}=\sum_{k=1}^{\infty} \frac{k+1}{(k \delta)^{\beta}}<\infty
$$

for each $\beta>2$, whereas the series diverges for $\beta=2$. Thus the convergence exponent $\rho_{1}=2$, and it follows from Lemma 2 that $f$ has order $\rho \geq 2$. But $\rho \leq 2$ by Lemma 1 . so we conclude that $\rho=2$. Since $\rho$ is a positive integer, Lindelöf's theorem applies.

For $m \delta<r \leq(m+1) \delta$, we calculate

$$
\begin{aligned}
S(r) & =\sum_{\left|z_{j}\right|<r} \frac{1}{z_{j}^{2}}=\sum_{k=1}^{m} \frac{1}{(k \delta)^{2}} \sum_{\ell=0}^{k} e^{-i \pi \ell / k} \\
& =\sum_{k=1}^{m} \frac{1}{(k \delta)^{2}} \frac{1+e^{-i \pi / k}}{1-e^{-i \pi / k}}=\sum_{k=1}^{m} \frac{1}{(k \delta)^{2}} \frac{\cos \frac{\pi}{2 k}}{i \sin \frac{\pi}{2 k}} \\
& \sim-\frac{2 i}{\pi \delta^{2}} \sum_{k=1}^{m} \frac{1}{k} \sim-\frac{2 i}{\pi \delta^{2}} \log m \sim-\frac{2 i}{\pi \delta^{2}} \log r
\end{aligned}
$$

as $r \rightarrow \infty$, which shows that the sums $S(r)$ are unbounded. By Lindelöf's theorem, this implies that $f$ has infinite type. However, this contradicts Lemma 1, which says that every function in $F_{\alpha}^{p}$ with $\rho=2$ has finite type $\tau \leq \frac{\alpha}{2}$. Therefore, $\Gamma$ cannot be the zero set of a function in $F_{\alpha}^{p}$.

Remark. The construction above relies on the fact that one can find interpolating sets for $F_{\alpha}^{p}$ whose exponent of convergence $\rho_{1}$ is exactly 2 . It is easy to see that every set $\Gamma=\left\{z_{j}\right\}$ with $\rho_{1}<2$ is an $F_{\alpha}^{p}$ zero set. In fact, the canonical product $P$ formed by $\left\{z_{j}\right\}$ has order $\rho=\rho_{1}<2$ (see Boas [1, p. 19). Thus $P \in F_{\alpha}^{p}$, and $\Gamma$ is an $F_{\alpha}^{p}$ zero set. The same argument shows that if $\Gamma$ is the zero set of some $f \in F_{\alpha}^{p}$ with order $\rho<2$, then $\rho_{1} \leq \rho<2$, so every subset of $\Gamma$ is also an $F_{\alpha}^{p}$ zero set.

\section{REFERENCES}

1. R. P. Boas, Entire Functions, Academic Press, New York, NY, 1954. MR0068627 (16:914f)

2. C. Horowitz, Zeros of functions in the Bergman spaces, Duke Math. J. 41 (1974), 693-710. MR0357747 (50:10215)

3. P. Duren and A. Schuster, Bergman Spaces, American Mathematical Society, Providence, RI, 2004. MR2033762 
4. K. Seip, Density theorems for sampling and interpolation in the Bargmann-Fock space I, J. Reine. Angew. Math. 429 (1992), 91-106. MR1173117 (93g:46026a)

5. K. Seip and R. Wallstén, Density theorems for sampling and interpolation in the BargmannFock space II, J. Reine. Angew. Math. 429 (1992), 107-113. MR.1173118 (93g:46026b)

6. K. Zhu, Zeros of functions in Fock spaces, Complex Variables Theory Appl. 21 (1993), 87-98. MR 1276563 (95b:30037)

Department of Mathematics, University of Michigan, Ann Arbor, Michigan 48104

E-mail address: ytung@umich.edu 